\title{
Natural Selection Under Conventional and Organic Cropping Systems Affect Root Architecture in Spring Barley
}

\author{
Md. Nurealam Siddiqui \\ University of Bonn \\ Michael Schneider \\ University of Bonn \\ Marissa B. Barbosa \\ University of Bonn \\ Jens Léon \\ University of Bonn \\ Agim Ballvora ( $\square$ ballvora@uni-bonn.de) \\ University of Bonn
}

\section{Research Article}

Keywords:

Posted Date: March 7th, 2022

DOI: https://doi.org/10.21203/rs.3.rs-1395532/v1

License: (c) (7) This work is licensed under a Creative Commons Attribution 4.0 International License. Read Full License 


\section{Abstract}

A beneficial root system is crucial for efficient nutrient uptake and stress tolerance. Therefore, it is critically important to evaluate the root system variation for breeding crop plants towards stress adaptation. We phenotyped root architectural traits of naturally adapted populations from organic and conventional cropping systems under hydroponic and field trails. Long-term natural selection under these two cropping systems resulted a microevolution in root morphological and anatomical traits. Barley lines developed under organic system possessed longer roots with narrow root angle, larger surface area, increased root mass density, and a thinner root diameter with an increased number of metaxylem vessels. In contrast, lines adapted to the conventional system tend to have a shorter and wider root system which possess a larger root volume with thicker diameter, but has fewer metaxylem vessels. Allometry analysis established a relationship between root traits and plant size among barley genotypes, which specify that root angle could be a good candidate among studied root traits to determine root-borne shoot architecture. Further, multivariate analyses showed a strong tendency towards an increased variability of the root morphological and anatomical traits in the organically adapted population. Our data indicates a significant differences in root phenotypes between conventional and organic populations, which could be useful in comparative genomics and breeding.

\section{Introduction}

Historically, crop improvement programs heavily focused on the role of above-ground plant functional traits for potential adaptation in specific abiotic stresses $^{1-3}$. While much research was performed on the above-ground parts of the plant, the below-ground part is often neglected, although their improvement is indirect in plant breeding programs ${ }^{4,5}$. Recently, the challenges in increasing food production with minimal environmental impact led to the renewed interest in understanding and improving the plant root system in terms of root architectural and anatomical attributes. The both root architectural and anatomical features are critical for plant endurance under climatic instability and nutrient insufficient conditions ${ }^{6-8}$.

The crucial role of roots for productivity and adaptability is dependent on roots system architecture- the collective term for the spatial and temporal arrangement of all root parts and root structural features arising from a single plant ${ }^{9}$. Root system architecture is dynamic and exhibits a high degree of plasticity in response to changing growing conditions such as soil moisture, soil structure, and composition, availability of nutrients, and the below-ground competition $^{4,9}$. The differences in root system architecture or root morphological characteristics were reportedly known to influence plants' competitive ability for soil resources, enabling them to respond and thrive in different agricultural systems ${ }^{9-12}$.

Barley (Hordeum vulgare L.) is a self-pollinating crop species under the Gramineae family and tribe Triticeae where two other evolutionary related grains crops wheat and rye, belonged ${ }^{13,14}$. Its importance was recognized since the start of civilization and was considered as one of the earliest grain crops in the world ${ }^{15}$. Now, barley is flourishing as a significant crop globally and ranks fourth after maize, wheat, and rice in terms of its area of cultivation and total production ${ }^{16}$. It is grown across several geographical regions worldwide, and because of its versatility and adaptability, this crop is successfully grown even in adverse agroecological conditions ${ }^{17}$. The crop is being utilized for it several essential uses, including; animal feed and fodder, malt production used in brewery and distillery industries, biofuels which is important as source of renewable energy ${ }^{18-21}$, and still remain as a staple food crop in some areas in the world, particularly in developing countries ${ }^{22}$.

In agricultural research, long-term selection experiments served as an important platform that could provide essential information on the performances and adaptability of plants as well as on the sustainability of the cropping systems ${ }^{23,24}$. While a number of studies investigated the impact of long-term selection experiments in some crops such as maize ${ }^{23}$, wheat ${ }^{25}$ and legumes ${ }^{24}$, these studies focus on the above-ground traits and highlighted on evaluating yield and yield stability, yield trends and sustainability, biomass productivity and nutrient cycling ${ }^{24}$. However, the effects of natural adaptation to different cropping systems, particularly allele frequency changes, has so far only been investigated in above-ground architecture. The effect of long-term adaptation on root system architecture and anatomy of economically important crop remains largely unknown and, in perspective of the potential of a low-input agriculture farming for sustainable crop production, further investigation is essential.

Root system architecture of barley populations modifies over time due to selection pressure by farming systems varying in the application of different types and amount of chemical fertilizers. Therefore, we hypothesize that barley populations, adapting twenty years towards organic cropping system (OCS) and conventional cropping system (CCS) establish variant root systems, promoted by the natural selection within the populations. To address this hypothesis, root phenotyping was performed on barley lines which were grown for twenty consecutive years in either an organically or conventionally managed cropping system. In this study, the major objective was therefore to combine and compare root anatomy and architecture responses and explore the effect of OCS and CCS on genetic variation in root traits. In details, we formulated the following objectives: (i) root-shoot phenotypic characterization of the CCS and OCS populations by investigating randomly selected subsets of these two naturally adapted lines, (ii) measure the root architectural traits in hydroponic environment where the entire root system extracted at the seedling stage, (iii) phenotype the root system traits attributes under field conditions, and (iv) compare the root morphology between CCS and OCS adapted lines to estimate the overall variation of root phenotypes.

\section{Materials And Methods}

Plant materials. Root phenotyping was performed in two barley $\mathrm{BC}_{2} \mathrm{~F}_{23}$ populations, originating from an identical founder population was developed by the Department of Plant Breeding, University of Bonn, Germany. The crossing scheme, and the cultivation practices were described by 26 and provided in Figure S1. In short, the initial barley population $\mathrm{BC}_{2} \mathrm{~F}_{3}$ was established by crossing the exotic line ISR42-8 (Hordeum vulgare ssp. Spontaneum), as donor and the modern cultivar Golf (Hordeum vulgare), as the recurrent parent. The establishment of the population was finalized by two rounds of back crossing with the parent Golf and two rounds of seed multiplication under controlled greenhouse conditions. The produced seeds of the $\mathrm{BC}_{2} \mathrm{~F}_{3}$ generation were used to establish the identical population under conventional and organic farming practice. Across the period of 20 generations, harvested seeds were used to establish next year 
population. In this process, the organic and conventional seed material was stored separately from another. We used the seeds from the organic environment to establish the next generation in the OCS environment and analogously proceeded in the CCS environment. No intended artificial selection was applied on the OCS and CCS populations across the entire 20 generations. Therefore, the cropping systems lead to changes in the allele frequency and with that also alters the phenotypic constitution of the populations. The natural selection process occurred under OCS and CCS for 20 generations (1999 to 2019 ) at faculty 's experimental station of Campus Klein-Altendorf, University of Bonn. The cultivation practices differed in the two farming systems as under organic conditions; a wide crop rotation was employed with no application of agrochemicals such as herbicides, pesticides and mineral fertilizers, while vice-versa under conventional conditions (Table S1A and S1B). In total, three hundred (300) and two hundred (200) lines were randomly selected for hydroponic and field root phenotyping, respectively. The experiments conducted both hydroponic and field conditions in accordance with relevant guidelines and regulations of Germany.

Hydroponic experiment. Three hundred barley lines, 150 evolved under long term selection in CCS and OCS each, were tested in a hydroponics experiment. The parental lines of these barley populations, Golf and ISR $42-8$ were randomly planted six times in each group. Prior to sowing, barley seeds were put in the oven for drying at $40^{\circ} \mathrm{C}$ for 24 hours to improve emergence rate. Two seeds per line were sown in each PVC tube ( $4.5 \mathrm{~cm}$ diameter $\mathrm{x} 45 \mathrm{~cm}$ depth) and both parental lines were randomly sown with three replications in each container. The seeds were germinated in situ in the PVC tubes contain Aquagran Filterquartz, 2-3.15 $\mathrm{mm}$ (Euroquarz $\mathrm{GmbH}$, Dorsten, Germany) with tap water. In case of both seeds germinating, one was selected for the experiment and the second plant was removed from the tube. The two groups were grown in separate hydroponic containers $(76 \mathrm{~cm} \times 59 \mathrm{~cm} \times 41 \mathrm{~cm})$, which were placed $80 \mathrm{~cm}$ above floor level next to each other. An additional container of the same size was used as a water and nutrient reservoir. A hydroponic water pump was used and immersed into these containers to periodically floods the plants with water and nutrients from the reservoir into the growing container through the hose connected between them. During planting, the reservoir boxes were filled with tap water and a day after, the containers were filled with balanced nutrient solution containing macro and micronutrients ${ }^{27}$. Then $\mathrm{pH}$ were adjusted to 5.9-6.0 using diluted $\mathrm{NaOH}$ and $\mathrm{HCl}$ and adjusted every second day. The water and nutrient solution were renewed once in a week to prevent nutrient exhaustion. Plants were grown in a greenhouse at the Campus Poppelsdorf, University of Bonn, Germany with $18 / 12^{\circ} \mathrm{C}$ temperature regime for 6 weeks at a photoperiod of $16 \mathrm{~h}$ supplemented with artificial lighting to maintain a minimum light intensity of $250 \mu \mathrm{mol}$ quanta $\mathrm{m}^{-2} \mathrm{~s}^{-1}$. Seedling emergence date was estimated in each genotype at 7 days after seed sowing.

Forty-five days after planting, plants were harvested for shoot and root measurements. Each tube was taken singly from the growing box and shaken gently to remove the grow substrate without destroying the roots. The whole plant was carefully pulled out from the tube and shoot measurements were manually performed. These include number of tillers and number of leaves, shoot height (which was measured from the root-shoot junction to the tip of the longest fully expanded leaf), and root length - measured from the hypocotyl to the root tip were gathered using a metric ruler. At approximately $0.5 \mathrm{~cm}$ from the root-shoot junction, the shoots were cut to separate the roots for further measurements.

Field experiment. The field evaluation was conducted from March to June 2020 at Campus Klein-Altendorf research facility (50³70' N, $\left.6^{\circ} 590^{\prime} \mathrm{E}\right)$, University of Bonn, Germany. The experiment was conducted under rainfed conditions on a homogen Luvisol with a high field capacity (approximately $25 \%$ ) and cation exchange capacity (CEC). The genotypes (individual lines within the population) from each of the two different populations were used for the field experiment. Both parental genotypes, Golf (cultivated variety) and ISR $42-8$ (wild-type) were included as respective control of each population derived under CCS and OCS. The experiment was carried out in a trial measuring $18 \mathrm{~m} \times 12.6 \mathrm{~m}$. This was divided into several plots, where each plot size was $1.5 \mathrm{~m} \times 1.12 \mathrm{~m}$. Each of these was subdivided into six rows, the barley cultivar Scarlett was sown in the two border rows, while ten seeds of each line were sown in each inner four rows. The plant's distance within a row was $14.5 \mathrm{~cm}$, while the distance between rows $21 \mathrm{~cm}$. The two population groups were grown in different sub nurseries, and 2 columns of plots planted with Scarlett were used as the borders to separate the two groups and the borders of the experimental area. No additional fertilizer was applied to the trial, with on demand pest control applied whenever necessary.

Roots were analyzed using "Shovelomics" approach ${ }^{12,28}$, followed by manual phenotyping. Three representative plants of more or less similar in shoot architecture in each line were selected and harvested at the complete flowering stage BBCH51 (Biologische Bundesanstalt, Bundessortenamt und CHemische Industrie ${ }^{29}$. In order to remove the soil debris, roots were rinsed with clean water to further remove remaining soil particles. Root angle $\left(^{0}\right)$ was determined as the angle between the two outermost seminal roots from the main shoot were then measured by a phenotyping board with a large protractor ${ }^{12}$. Afterwards, root samples were preserved in $70 \%$ alcohol $(\mathrm{v} / \mathrm{v})$ for image acquisition and morphological trait quantification.

Root image acquisition and processing using WinRHIZO. To quantify root morphological traits, root samples stored in $70 \%$ alcohol were placed in a plexiglass scanner tray $(20 \mathrm{~cm} \times 30 \mathrm{~cm})$ with a 3 to $4 \mathrm{~mm}$ deep layer of water. They were adjusted to help untangle the roots and minimize overlapping and aligned vertically on the scanning plates. Thereafter, roots were scanned with a high-resolution Epson scanner (Perfection LA24000) at 600 dots per inch, giving an eight-bit grayscale image. The entire root system rather than a subsample of the root was scanned to avoid errors associated during root sampling. The captured images were subsequently used for root analysis which was performed using WinRHIZO Regular (version 2020a, Regent Instruments Inc., Quebec, Canada). The WinRHIZO software's output gave the following measurements: root length (RL), root volume (RV), root average diameter (RAD), root surface area (RSA) and number of root tips and forks. The variables calculated as described by ${ }^{30}$ were as follows: root tissue mass density (RMD) as the ratio of RV and root dry weight (RDW). After subsequent analysis, root samples were then oven-dried at $70^{\circ} \mathrm{C}$ for $48 \mathrm{~h}$ to record the RDW. Trait description with acronyms and unit are provided in Table 1.

Root anatomical study. To analyze root anatomical parameters, nodal root parts of the main shoot axis at approximately $5-7 \mathrm{~cm}$ from the root-shoot junction were selected. The selected root samples were then fixed in $70 \%(\mathrm{v} / \mathrm{v})$ alcohol until further analysis. Cross-section slides were made by cutting the fixed samples by hand with a sharp razor blade (Apollo, HERKENRATH Solingen, Germany). Thereafter, cuttings were placed on a glass slide, and root cross-section images were taken using a digital microscope (Keyence's VHX-1000D, Germany) with 100X magnification. At least three to five root images per genotype were considered for measuring root anatomical traits. Acquired images were then analyzed by ImageJ software ${ }^{31}$, which measured anatomical parameters such as 
root cross-section area (RXA), total cortical area (TCA), stele area (SA), aerenchyma area (AA), late metaxylem number (LMXN) and average late metaxylem area (ALMXA). Trait description with acronyms and unit are provided in Table 1.

Statistical analysis. Statistical analyses were performed using R version 4.0.1 (R core development team). For data obtained under hydroponics growing conditions, one-way ANOVA was used to evaluate phenotypic differences between the OCS and CCS populations and their parental lines. Means in each group were compared based on Tukey's HSD using R package agricolae version 1.3-3 at 95\% family-wise confidence interval. Pearson correlation analysis was performed on corresponding morphological traits between the two groups under the two growing conditions using 'corrplot' package in R studio. Additionally, an allometric test for the similar tissue development was performed by the 'npregfast' R package ${ }^{32}$. A principal component analysis (PCA) using 'PCAtools' 33 in $\mathrm{R}$ package version 2.0.0 was performed to access the phenological diversification between both CCS and OCS. Box- and density plots were generated with the package 'ggpubr' (version 0.4.0), where the compare means extension was implemented as a simple t-test to compare the groups.

\section{Results}

Root morphological traits. The wild-type parent ISR42-8 produced longer root length (RL) than the modern cultivar parent Golf and tested lines (Table S2, Fig. 1A.h, 1A.f [h=hydroponic; $f=f i e l d]$ ). The tested lines of the two evolving barley populations displayed significant variations under hydroponic conditions. Barley lines evolved under OCS had on average $3484 \mathrm{~mm}$ longer roots compared to CCS under hydroponic treatment (Fig. 1A.h, Table 2). Complementary results under field conditions show as well higher RL for the OCS lines, even though the variance was significantly less pronounced (Fig. 1A.f). In addition, less evident variance was observed in field within both groups compared to the hydroponic (Fig. 1A.d). Across both experimental setups, the observed range of RL was higher in the OCS lines [Standard deviation $(\mathrm{SD})_{\mathrm{OCS}}=883, \mathrm{SD}_{\mathrm{CCS}}=597$ ] (Table 2).

The root length to volume (L/V) is an important indicator of the soil volume that can be explored by the roots. Under hydroponics conditions, variations were found for L/V between the parental genotypes as well as between the OCS and CCS populations (Table 2 \& S2). The organic lines were characterized by a significantly higher L/V, indicating a much more distinct exploration of the soil by these lines (Fig. 1B.h, 1B.f). In comparison to field, highest diversity in L/V was found under hydroponic experiments within both OCS and CCS populations (Fig. 1B.d).

The root mass density (RMD) is the ratio of root volume for a given root mass and is a key indicator of root thickness. Although significant variations existed between ISR42-8 and Golf under hydroponics conditions, such significant variations were not found between the OCS and CCS groups $(P=0.09)$ (Fig. 1C.f and Table 2 \& S2).

The root angle (RA) measurements were only performed under field conditions, since plants grown under hydroponics conditions were placed in uniform growing vessels and direction of root growth is restricted by tubes. Significant variation was observed for the RA between the two parental lines, which was also reflected in the CCS and OCS lines (Fig. 1D.f). ISR42-8 was characterized by an $11.5^{\circ}$ average narrower RA than Golf (Table S2). The RA was $4.1^{\circ}$ bigger in the OCS compared to the CCS population $(P=0.005)$ (Table 2). However, a higher diversity in RA was observed in the OCS compared to the CCS lines (Fig. 1D.d, Table 2).

In addition to the RA, the number of root tips and forks were measured under field conditions only. Both tips and forks indicate a similar pattern, where the OCS lines produced on average more for both $P_{\text {Forks }}=0.014, P_{\text {Tips }}=0.0041$ (Fig. 1E.f, 1F.f). After applying a $P$-adjustment, the number of forks count was no longer significantly different between OCS and CCS $\left(P_{\text {Forks }}=0.07\right.$, Table 2). Complementary, ISR 42-8 was observed to produce more tips and forks than Golf, which remained highly significant even after probability adjustment (Fig. 1E.f, 1F.f, Table S2). The distribution and the standard deviation of observed phenotypes highlights once more the fact that the OCS lines tend to have a higher variation (Fig. 1E.d, Table 2). Similarly, significant increasing trend was recorded root surface area (RSA) and root average diameter (RAD) by ISR42-8 as compared to Golf under hydroponics (Table S2). Contrasting to the parental genotypes, no variation was observed between OCS and CCS lines for RSA (Table 2).

Root anatomical traits. Within the observed anatomical traits, four were considered due to their relevance and variation between the systems. In both hydroponic and field experiment, significant variations were observed for the late metaxylem number (LMXN) between the parental lines as well as OCS and CCS lines (Table 2 \& S2, Figs. 2A.h, 2A.f). An increased LMXN for ISR 42-8 compared to Golf was observed (Fig. 2A.h). In regard to the CCS and OCS lines, a heterogenic scenario was presented over both experimental setups. While the median LMXN under CCS was identical with ISR $42-8$ in the seedling stages of plant development (Fig. 2A.h), it was much lower in flowering stages under field conditions (Fig. 1A.f). Additionally, the LMXN was significantly higher in the CCS lines in seedling stage compared to OCS lines, vice-versa LMXN was observed at flowering time point (Fig. 2A.d).

The intercellular space, represented by aerenchyma area (AA), was observed to be significantly more pronounced in the tested CCS compared to OCS lines in both environments (Fig. 2B.h, 2B.f). Furthermore, the OCS population did not show significant differences to both parents under hydroponics conditions, however, when grown under field conditions, it was noted that Golf had significantly higher AA mean value as compared to the OCS population (Table S2). As illustrated by the values, the AA expended from early to late stages by a magnitude of 10 -folds (Fig. 2B.d). In general, although the two parents did not indicate phenotypic variations, OCS and CCS lines showed significant variations (Table S3).

A 0.12 lower average total cortical rea (TCA) was recorded in the OCS compared to the CCS population under field conditions $(P=0.003$, Fig. 2C.f), although substantial variations for TCA was observed within OCS and CCS populations (Fig. 2C.d). The root cross-section area (RXA) is a two-dimensional axis of the root which is an important indicator of root thickness. In the hydroponic examination of the seedling stage, significant variations existed between the CCS and ISR42-8 as well as OCS population (Table 2 and S2). The complementary study under field conditions observed a noticeable variation for OCS from both parental genotypes and the CCS (Table S2). About $0.13 \mathrm{~mm}^{2}$ increased average value for RXA was identified for CCS (Fig. 2.D.f), while consistent significant variations were also observed between the populations under field experiment (Fig. 2D.d). Analogue to the AA, the RXA indicates a lower root extension in the

Page $4 / 13$ 
OCS compared to the CCS population. For the stele area (SA), significant variations were only observed at flowering stage, where ISR42-8 generally had the highest SA and varies significantly between Golf and to its progeny lines (Table 2 and S2).

Shoot-related traits. Beyond the root-related phenotypic observations, above-ground characteristics were also recorded to assess the root-borne shoot dynamics (Fig. 3 and S2). Among the OCS and CCS populations and the parents, ISR42-8 had the longest duration of emergence. While CCS adapted lines took on average 5.8 day of emergence (DE), OCS adapted lines emerged 1.8 days later (7.6 days) (Fig. S2). No variation was observed for the tiller number (TN) throughout all tested groups, while ISR $42-8$ tends to produce much more leaf number (LN), accompanied by a lower plant height (PH) and higher shoot dry weight (SDW) (Table S3, Fig. S2). The OCS and CCS plants significantly differed in PH as well as SDW (Fig. 3B, 3C). The LN was marginally above the probability threshold of $0.05(p=0.058$, Fig. 3A), with a clear tendency of increased variability in phenotypic variation (Fig. 3D). Similar trend was recorded for the SDW (Fig. 3F).

Interconnection of root-shoot traits. We performed inter-trait correlation analysis to unravel association among root traits and in between root and shoot phenotypes (Fig. 4). Pearson correlation coefficient revealed significant correlations among root-shoot traits. LN, PH and SDW had strong positive associations with all root architectural traits under hydroponic conditions $(P<0.001, r=>0.30)$ in both CCS and OCS, while DE has negative association with all shoot traits $(r=-0.17$ to -0.48$)$ (Figs. 4A.h and 4B.h). A consistent negative relationship was observed for L/V with shoot traits such as LN, PH and SDW and root morphological traits such as RL, RSA and RAD in both CCS and OSC populations (Figs. 4A.h and 4B.h). A strong negative association existed between RL and all shoot morphological, root architectural and anatomical traits in both populations, except for L/V where a weak negative $(r=-0.09)$ association was displayed only in the OCS. Likewise, all above-ground traits and all root architectural traits exhibited significant positive associations to all root anatomical features in both groups with an exception for the AA (Figs. 4A.h and 4B.h). Moreover, correlation analysis revealed strong positive relation in both groups of SDW and root dry weight (RDW) to all above-ground traits, below-ground traits including, RL, SA and RAD, as well as in all root anatomical traits (Figs. 4A.h and $4 \mathrm{~B} . \mathrm{h}$ ). This means that the growth of tissue and organ is proportional to the increase to total dry biomass. More importantly, we observed a significant positive correlation among most of the root morphological, architectural and anatomical traits in both OCS and CCS adapted populations, with few exception such as L/V (Figs. 4A.h and 4B.h).

Similar to hydroponics, correlation analysis for both groups under field conditions showed highly significant positive and negative correlations (Figs. 4A.f and 4B.f). While a positive correlation was observed under hydroponics, RDW only showed to be positive significant relation with other root morphological traits and a weak positive relationship with anatomical traits in response to both CCS and OCS. Interestingly, correlation analysis revealed the important association of RA to other root architectural and anatomical features, showed strong negative association to RL ( $r=>-0.90)$ and RDW ( $r=>-0.80)$ (Figs. 4A.f and 4B.f) for CCS and OCS populations respectively, which means that narrower the angle of the nodal roots, the longer was the root system. The two root branching traits, number of tips and number of roots forks which were known to be associated and dependent on the RL to have a strong positive correlation reflected by $r=$ 0.81 and 0.90 in CCS and r $=0.74$ and 0.84 in OCS developed lines, respectively, while they have significant negative correlation with RA ( $r=-0.72$ to -0.77 ) in both barley groups. In addition, RA had also strong negative relationship to RDW contributing architectural traits including, RMD ( $r=-0.81$ to -0.84$)$ and $L / V(r=$ -0.34 to -0.44 ). However, no positive associations were observed for RA and all root anatomical traits in both OCS and CCS populations (Figs. 4A.f and 4B.f).

Allometry analysis. The correlation analysis identified interconnection among root and shoot-related traits. Therefore, we checked if these correlations can be explained by allometric relations (Table 3 and 4 ).

In the hydroponic environment, we observed a total of ten allometric relations, from which six were annotated to the $\mathrm{PH}$. The $\mathrm{PH}$ was allometrically related to the SDW, the RSA, the RV, the RDW, the SRL, and the RMD (Table 3). Besides, the SDW was allometrically associated with the RSD. Furthermore, the TCA was related to the RXA. Finally, an allometry relationship was detected between the LMXN and AA (Table 3).

In the field experimental set up, we detected in total ten allometric relations $(P<0.05)$. The most significant allometry was observed for the RL and the RA (Table 4). Besides, the RA was allometrically related to the RDW, the SRL, the RMD and the number of root forks. Furthermore, the RAD growth was significantly related to the growth of RSA. Finally, we also observed an allometric relationship between TCA and the SA (Table 4).

Root system divergence exists in barley populations. Based on the root phenotypic traits, PCA was conducted to highlight variations between parental lines as well as OCS and CCS populations (Fig. 5). Lines evolved in the OCS environment tend to have a higher variance compared to CCS adapted lines in both experimental setups (Hydroponics and field, Figs. 5A and 5B). The majority of variance is explained by the first component (PC1) (81.71\% hydroponics, $97.46 \%$ field), while the second component (PC2) explains $18.28 \%$ and $2.52 \%$ for hydroponics and field experiment, respectively (Fig. 5). The principal components (PCs) of both environments highlight a similar range of values and extension of points.

\section{Discussion}

Here we report that after 20 years of judicious natural adaption towards OCS and CCS, barley populations segregated in root phenotypes. Variation of root system architecture is regarded as a significant aspect of plants' adaptability in response to fluctuating growing conditions ${ }^{5,12,34,35}$. These differences are critical parameters that could influence plants' competitive ability in searching for unevenly distributed soil resources and hence a predictor of plant's adaptation to various growing conditions $9,10,36$.

Trait evaluations were performed in two different test environments (hydroponic and field) with the intention to produce redundant and additional phenotypic information. This resulted in a better resolution for some root traits under both hydroponics and field experiments. We performed the experiment without additional replications of each line, as the overall objective was to determine phenotypic variation between the CCS and OCS populations and not between the genotypes within the populations. Both organic and conventional genotypes grown under hydroponics and field conditions were harvested identically, which results in an identical bias among the groups. Nevertheless, the testing under field condition could result an increased underestimation of root architectural 
parameters, therefore we complemented the field trial with a hydroponic experiment, where the entire root system morphology could be estimated. As already shown in root morphological trials in Brassica, the root development in seedling stage is a reliable indicator for the following development stages ${ }^{37}$. We hypothesized that this is also true in barley.

Another important reason that could be pointed with the differences among root parameters acquisited in both growing conditions is the variable access of resources needed for plant growth. Equal and consistent access to water, nutrients, oxygen, and other physical factors such as temperature and light were provided continuously under hydroponics growing conditions whereas, plants are exposed to fluctuating environment factors, and competition of soil resources occurs when they are grown under field condition. These factors were reported to directly or indirectly influence root growth ability and development ${ }^{38}$. The two parental genotypes used to establish the populations are highly variant in terms of their root architecture ${ }^{39}$. While ISR $42-8$ is a wild genotype, adapted to its local arid low input environment, Golf is a former elite cultivar, selected to produce high yields in humid climates under nutrient supplementation. These two divergent genetic backgrounds were partly reflected in the phenotypes of the subset of the lines selected from OCS and CCS populations (Figs. 1 and 2). While CCS were supplemented with mineral fertilizers and therefore not exposed to an environment of nutrient deficiency, the OCS population was not supplemented with mineral fertilizer (Table S1B). We observed that the variations in RL, L/V and RA in the OCS population was more pronounced compared to the CCS (Fig. 1A-C). Alike to the RL, the increase of RL by the OCS line is likely associated with the genetic background of ISR $42-8$, so it could be assumed that the root grows towards deep soil layers to capture deep soil resources ${ }^{6,40,41}$. Deep and steeper roots leads to improved access of soil resources and consequently improves yield in some crops such as rice ${ }^{42}$, wheat ${ }^{43}$, and nitrogen uptake in maize ${ }^{44}$. Moreover, longer roots with narrow RA, higher RMD and L/V, and bigger RSA were recorded among lines originated from OCS (Fig. 1; Table 2). This data, especially from the hydroponic experiment, indicated that these lines potentially target deep soil layers. The results presented are substantiated by the recent study ${ }^{45}$. They evaluated the early seedling traits of evolving winter wheat composite cross populations (CCPs) and reported that overtime CCPs exposed to the organic management system for several generations $\left(F_{11.1}\right)$ showed considerably higher root performance as displayed by its longer seminal RL, narrow system and higher RDW.

Important features of root anatomical adjustments between lines originated in two different management systems were significantly variant, mostly recorded in LMXN, TCA, AA and RXA (Fig. 2). RXA is mainly associated with root thickness, which is relatively expressed in the subsequent thickness of the root cortical and pith tissue layers ${ }^{46}$. It is also well reported that the thick root diameter is associated with large cortex and aerenchyma size ${ }^{47,48}$. In this investigation, barley lines that produce a bigger RAD also have bigger RXA, TCA, and AA (Table 2, Fig. 2). Likewise, conventionally selected lines that were found to have bigger RAD and RV compared to those under the organic system indeed also produce comparatively bigger size for root anatomical traits such as RXA, ALMXA, TCA, AA, and SA than barely lines evolved under the organic system (Table 2). Comparing the two barley populations, organically managed lines had superior LMXN than conventionally managed lines (Figs. 2A,h and 2A,f).

Our results indicated that barley lines with thinner roots due to decreased TCA or SA and had a smaller ALMXA and tend to have a LMXN, which particularly displayed by lines evolved under the OCS. This might indicate a variation in the development of these root anatomical organs between the cropping systems. The effect could either result from a long-term farming practice-depended variation or from the exposed environment itself. Under hydroponic conditions, water supply was never in shortage, while it is a more limiting factor under field conditions. Therefore, the observed patterns could be an indication of increased metaxylem size, which ultimately is a reflector of water stress response ${ }^{49}$. In contrast, the higher LMXN in the OCS lines might be an indication of less lines suffer from water limitations. A relevant correlation between the RL and the LMXN could not be observed, most likely due to the impossibility of harvesting the root as a whole under field conditions. However, several reports noted that water-conducting tissue tend to be smaller, plants tend to develop more LMXN to compensate smaller diameters ${ }^{48,50,51}$. Narrow xylem diameter, particularly in seminal roots, was reported to optimize soil water from deep soil profile for plant utility, especially during maturation ${ }^{50}$, whereas broad xylem diameter in deep-growing roots were known to also improve the exploration of available water within deep soil horizons ${ }^{4,52}$. The cortex, which is composed of parenchyma tissue, plays a crucial role in regulating water and nutrient transport via the apoplast and symplast pathways ${ }^{53}$. A lower TCA was recorded by OCS (Fig. $2 \mathrm{C}$, f) that might be associated with a higher nutrient and energy use efficiency. As OCS lines already tend to produce longer RL, a thick cortical area would extend their fitness disadvantages in terms of energy allocations away from the seeds ${ }^{54}$. Moreover, aerenchyma (air cavities that consist of gas-filled spaces) plays a vital role in the long-distance transport of oxygen from the shoots to the root tips which is particularly important in the adaptability and survival of plants particularly under stressful growing condition ${ }^{51}$. Root aerenchyma has been related to improving crop growth in maize plants grown under water-deficit conditions ${ }^{52,55}$.

Additionally, allometric association were observed between root traits and plant size. The most relevant is the RA to RL, RDW, and number of root forks (Tables 3 and 4). These traits, especially RA might be a good candidate for the root breeding as it determines the entire root system development. Nevertheless, the genetic background of all lines is limited to two inbreed lines, making extrapolations of such allometric observations difficult. Besides the RA, only the $\mathrm{PH}$ indicated six allometric relations. This might be associated with the rapid growth in the early growth stage and therefore, the allometry of the $\mathrm{PH}$ might be overestimated. Moreover, an increased phenotypic variation was observed in the OCS lines over various root morphological and anatomical traits (Fig. 5 and Table S3). This heterogeneity might originate from genotypes inhabit different ecological niches within the population, e.g. some genotypes might have more shallow roots to capture the upper soil layers, while others have predominantly developed higher $\mathrm{RL}$ to reach deep soil layers ${ }^{41}$.

In conclusion, the tested lines from the organic adapted population were observed to have an adaptive root system, represented by longer roots with a narrow root angle, larger root surface area, root density and a thinner root size with higher late metaxylem number. In comparison to the organic population, lines adapted to conventionally treated lines tend to have a shorter and wider root architecture, which maintain a larger root volume and thicker root size but has fewer late metaxylem number (Figs. 1 and 2). Moreover, long-term selection by organic farming system contributed substantial genetic variation in root system attributes observed under both hydroponic and field conditions (Fig. 5). The deep rooting patterns of organic lines can be beneficial in terms of soil erosion reduction ${ }^{56}$, drought tolerance ${ }^{57,58}$, lodging tolerance ${ }^{59}$, and increased nutrient use efficiency and preserved leaching ${ }^{60}$. Moreover, our results indicate 
different requirement of root growth between OCS and CCS, which may provide an additional insight into the nutrient use efficiency. Varieties with the described root phenotypes could serve as valuable genetic resources in future breeding.

\section{Declarations}

\section{Acknowledgements}

The authors gratefully acknowledge German Academic Exchange Service (DDAD) and Erasmus Mundus Master Program in Plant Breeding (emPLANT) for providing fellowship to MNS and MB, respectively. The authors also grateful to Karola Müller and the colleagues Campus Klein-Altendorf of Bonn University for their technical support.

\section{Author contributions}

J. L. and A. B. conceived and supervised the study. M.N.S. and M.S. performed the experiments, data analysis, writing and editing the manuscript. M.B.B. performed the experiments and generated data. J.L. and A.B. reviewed and edited the manuscript. All authors have read and approved the manuscript.

\section{Data availability}

The authors confirm that the data supporting the findings of this study are available from the corresponding author, upon request.

\section{Conflict of interest}

The authors declare no conflict of interest.

\section{References}

1. Maes, S.L., Perring, M.P., Depauw, L., Bernhardt-Römermann, M. et al. Plant functional trait response to environmental drivers across European temperate forest understorey communities. Plant Biol. 22 (3): 410-424 (2020).

2. Meister, R., Rajani, M.S., Ruzicka, D., Schachtman, D.P. Challenges of modifying root traits in crops for agriculture. Trends Plant Sci. 19 (12): $779-788$ (2014).

3. Zhang, J., Li, P. Response of plant functional traits to climate change. IOP Conf Ser: Earth Environ. Sci. 300 (3): 032078 (2019).

4. Lux, A., Rost, T.L. Plant root research: the past, the present and the future. Ann. Bot. 110 (2): 201-204 (2012).

5. Siddiqui, M.N., Léon, J., Naz, A.A., Ballvora, A. Genetics and genomics of root system variation in adaptation to drought stress in cereal crops. J. Exp. Bot. 72 (4): 1007-1019 (2021).

6. Del Bianco, M., Kepinski, S. Building a future with root architecture. J. Exp. Bot. 69 (22): 5319-5323 (2018).

7. Paez-Garcia, A., Motes, C.M., Scheible, W.R. et al. Root traits and phenotyping strategies for plant improvement. Plants 4(2): 334-355 (2015).

8. Purushothaman, R., Zaman-Allah, M. et al. Root anatomical traits and their possible contribution to drought tolerance in grain legumes. Plant Prod. Sci. 16 (1): 1-8 (2013).

9. Lynch, J. Root architecture and plant productivity. Plant Physiol. 109(1): 7 (1995).

10. Ingram, P.A., Malamy, J. Root system architecture. Adv. Bot. Res. 55: 75-117 (2010).

11. Malamy JE (2005) Intrinsic and environmental response pathways that regulate root system architecture. Plant Cell Environ 28(1), 67-77.

12. Oyiga, B.C. Palczak, J., Wojciechowski, T. et al. Genetic components of root architecture and anatomy adjustments to water-deficit stress in spring barley. Plant Cell Environ. 43(3): 692-711 (2020).

13. Friedt, W., Horsley, R. D., Harvey, B. L., Poulsen, D. M. E., Lance, R. C. M., Ceccarelli, S. et al.. Barley breeding history, Progress, Objectives, and Technology. In S.E. Ullrich (Hrsg.), Barley (1. Auflage, S. 160-220) (2010).

14. El-Hashash, E. F., \& El-Absy, K. M. Barley (Hordeum vulgare L.) breeding. In Advances in Plant Breeding Strategies: Cereals (pp. 1-45). Springer, Cham (2019).

15. Morrell, P.L., Clegg, M.T. Genetic evidence for a second domestication of barley (Hordeum vulgare) east of the Fertile Crescent. PNAS 104: $3289-94$ (2007).

16. FAO. World Food and Agriculture - Statistical Pocketbook 2018. Rome. 254 pp. Licence: CC BY-NC-SA 3.0 IGO (2018).

17. Zhou, M. X. Barley Production and Consumption. In G. Zhang \& C. Li (Hrsg.), Genetics and Improvement of Barley Malt Quality (S. 1-17). Berlin, Heidelberg: Springer Berlin Heidelberg doi: 10.1007/978-3-642-01279-2 (2009).

18. Pomeranz, Y., Shands, H.L. Food uses of barley. CRC Critical Rev. Food Tech. 4: 377-394 (1974).

19. Cowan, W.D., Mollgaard, A. Alternative uses of barley components in the food and feed industries. In: Sparrow R C M, Lance, Henry R J (eds.) Alternative End Uses of Barley. DHB (1988).

20. Griffey, C., Brooks, W., Kurantz, M., Thomason, W. et al. Grain composition of Virginia winter barley and implications for use in feed, food and biofuels production. J. Cereal Sci. 51: 41-49 (2010).

21. Schwar, P., Li, Y. Malting and brewing uses of barley. In: Ullrich SE, editor. Barley: Production, Improvement, and Uses. Oxford: Wiley-Blackwell. pp. 478$521(2011)$. 
22. Tricase, C., Amicarelli, V., Lamonaca, E. \& Leonardo Rana, R. Economic Analysis of the Barley Market and Related Uses. In Z. Tadele (Hrsg.), Grasses as Food and Feed. IntechOpen. doi:10.5772/intechopen.78967 (2018).

23. Hirsch, C. N., Flint-Garcia, S. A., Beissinger, T. M., Eichten, S. R., Deshpande, S., Barry, K. et al.. Insights into the Effects of Long-Term Artificial Selection on Seed Size in Maize. Genetics 198 (1): 409-421 (2014).

24. Wortman, S.E., Galusha, T.D., Mason, S.C., Francis, C.A. Soil fertility and crop yields in long-term organic and conventional cropping systems in Eastern Nebraska. Renew Agric. Food Syst. 27(3): 200-216 (2012).

25. Armengot, L., Berner, A., Blanco-Moreno, J.M. et al. Long-term feasibility of reduced tillage in organic farming. Agron. Sustain. Dev. 35: $339-346$ (2015).

26. Schneider, M., Barbosa, M., Ballvora, A., Leon, J. Organic farming-deep genotyping reveals specific selection footprints in barley populations. doi: 10.21203/rs.3.rs-266048/v1 (2021).

27. Hoagland, D.R., Snyder, W.C. Nutrition of strawberry plant under controlled conditions. (a) Effects of deficiencies of boron and certain other elements, (b) susceptibility to injury from sodium salts. Proceed.Ameri. Soc. Hort. Sci. 30: 288-294 (1933).

28. Trachsel, S., Kaeppler, S.M., Brown, K.M., Lynch, J.P. Shovelomics: high throughput phenotyping of maize (Zea mays L.) root architecture in the field. Plant Soil 341(1): 75-87 (2011).

29. Meier, U. Growth stages of mono-and dicotyledonous plants. Blackwell Wissenschafts-Verlag (1997).

30. Nakhforoosh, A., Grausgruber, H., Kaul, H.P., Bodner, G. Wheat root diversity and root functional characterization. Plant Soil 380(1): 211-229 (2014).

31. Schneider, C.A., Rasband, W.S., Eliceiri, K.W. NIH Image to ImageJ: 25 years of image analysis. Nat. Methods 9(7): 671-675 (2012).

32. Sestelo, M., Villanueva, N.M., Meira-Machado, L., Roca-Pardiñas, J. npregfast: An R package for nonparametric estimation and inference in life sciences. J. Stat. Softw. 82(1): 1-27 (2017).

33. Blighe, K. PCAtools: PCAtools: Everything principal components analysis. R package version 1.1. 10 (2019).

34. Kadam, N.N., Tamilselvan, A., Lawas, L.M., Quinones, C., Bahuguna, R.N., Thomson, M.J. et al. Genetic control of plasticity in root morphology and anatomy of rice in response to water deficit. Plant Physiol. 174(4): 2302-2315 (2017).

35. Sultan, S.E. Phenotypic plasticity in plants: a case study in ecological development. Evol. Dev. 5(1): 25-33 (2003).

36. Osmont, K.S., Sibout, R., Hardtke, C.S. Hidden branches: developments in root system architecture. Annu. Rev. Plant Biol. 58: 93-113 (2007).

37. Louvieaux, J., Spanoghe, M., Hermans, C. Root morphological traits of seedlings are predictors of seed yield and quality in winter oilseed rape hybrid cultivars. Front. Plant Sci. 11. doi: 10.3389/fpls.2020.568009 (2020).

38. Boyer, J.S. Plant productivity and environment. Science 218(4571): 443-448. doi: 10.1126/science.218.4571.443 (1982).

39. Naz, A.A., Arifuzzaman, M., Muzammil, S., Pillen, K., Léon, J. Wild barley introgression lines revealed novel QTL alleles for root and related shoot traits in the cultivated barley (Hordeum vulgare L.). BMC Genet. 15(1): 107 (2014).

40. Hochholdinger, F., Tuberosa, R. Genetic and genomic dissection of maize root development and architecture. Curr Opin. Plant Biol. 12(2): 172-177 (2009).

41. Thorup-Kristensen, K., Halberg, N., Nicolaisen, M., Olesen, J.E., Crews, T.E., Hinsinger, P. et al. Digging deeper for agricultural resources, the value of deep rooting. Trends Plant Sci. 25(4): 406-417 (2020).

42. Liu, K., He, A., Ye, C., Liu, S., Lu, J., Gao, M. et al. Root morphological traits and spatial distribution under different nitrogen treatments and their relationship with grain yield in super hybrid rice. Sci. Rep. 8(1): 1-9 (2018).

43. Manschadi, A.M., Christopher, J., deVoil, P., Hammer, G.L. The role of root architectural traits in adaptation of wheat to water-limited environments. Funct. Plant Biol. 33(9): 823-837 (2006).

44. Mi, G., Chen, F., Wu, Q., Lai, N., Yuan, L., Zhang, F. Ideotype root architecture for efficient nitrogen acquisition by maize in intensive cropping systems. Sci. China Life Sci. 53(12): 1369-1373 (2010).

45. Vijaya, V.B., Baresel, J.P., Weedon, O., Finckh, M.R. Effects of ten years organic and conventional farming on early seedling traits of evolving winter wheat composite cross populations. Sci. Rep. 9(1): 1-12 (2019).

46. Yamauchi, T., Abe, F., Tsutsumi, N., Nakazono, M. Root cortex provides a venue for gas-space formation and is essential for plant adaptation to waterlogging. Front Plant Sci. 10: 259(2019).

47. Bramley, H., Turner, N.C., Turner, D.W., Tyerman, S.D. Roles of morphology, anatomy, and aquaporins in determining contrasting hydraulic behavior of roots. Plant Physiol. 150 (1): 348-364 (2009).

48. Hummel, I., Vile, D., Violle, C., Devaux, J., Ricci, B. et al. Relating root structure and anatomy to whole-plant functioning in 14 herbaceous Mediterranean species. New Phytol. 173(2): 313-321 (2007).

49. Kadam, N.N., Yin, X., Bindraban, P.S., Struik, P.C., Jagadish, K.S. Does morphological and anatomical plasticity during the vegetative stage make wheat more tolerant of water deficit stress than rice? Plant Physiol. 167(4): 1389-1401 (2015).

50. Lynch, J.P., Chimungu, J.G., Brown, K.M. Root anatomical phenes associated with water acquisition from drying soil: targets for crop improvement. J. Exp. Bot. 65(21): 6155-6166 (2014).

51. Phule, A.S., Barbadikar, K.M., Madhav, M.S., Subrahmanyam, D. et al. Studies on root anatomy, morphology and physiology of rice grown under aerobic and anaerobic conditions. Physiol. Mol. Biol. Plants 25(1): 197-205 (2019).

52. Zhou, Y., Watts, S.E., Boutton, T.W., Archer, S.R. Root density distribution and biomass allocation of co-occurring woody plants on contrasting soils in a subtropical savanna parkland. Plant Soil 438(1): 263-279 (2019).

53. Steudle, E., Peterson, C.A. How does water get through roots? J. Exp. Bot. 49(322): 775-788 (1998). 
54. Colombi, T., Herrmann, A.M., Vallenback, P., Keller, T. Cortical cell diameter is key to energy costs of root growth in wheat. Plant Physiol. 180(4): 20492060 (2019).

55. Bouranis, D.L., Chorianopoulou, S.N., Siyiannis, V.F., Protonotarios, V.E., Hawkesford, M.J. Aerenchyma formation in roots of maize during sulphate starvation. Planta 217(3): 382-391 (2003).

56. Vannoppen, W., De Baets, S., Keeble, J., Dong, Y., Poesen, J. How do root and soil characteristics affect the erosion-reducing potential of plant species?. Ecol. Eng. 109: 186-195 (2017).

57. Comas, L.H., Becker, S.R., Cruz, V.M.V., Byrne, P.F., Dierig, D.A. Root traits contributing to plant productivity under drought. Front. Plant Sci. 4. doi:10.3389/fpls.2013.00442 (2013).

58. Wasaya, A., Zhang, X., Fang, Q., \& Yan, Z. Root phenotyping for drought tolerance: A review. Agron. 8(11): 241(2018).

59. Karim, M.H., Jahan, M.A. Study of lodging resistance and its associated traits in bread wheat. J. Agric. Biol. Sci. 8(10): 683-687 (2013).

60. Li. X., Zeng, R., Liao, H. Improving crop nutrient efficiency through root architecture modifications. J. Integr. Plant Biol. 58(3): 193-202 (2016).

\section{Tables}

Table 1

List of studied and derived phenotypic traits with trait acronym and unit.

\begin{tabular}{|c|c|c|}
\hline Traits & Trait acronym & Unit \\
\hline \multicolumn{3}{|l|}{ (A) Shoot morphological traits } \\
\hline Date of emergence & $\mathrm{DE}$ & Days after sowing (DAS) \\
\hline Plant Height & $\mathrm{PH}$ & $\mathrm{cm}$ \\
\hline Tiller Number & $\mathrm{TN}$ & plant $^{-1}$ \\
\hline Leaf Number & $\mathrm{LN}$ & plant $^{-1}$ \\
\hline Shoot dry weight & SDW & g plant $^{-1}$ \\
\hline \multicolumn{3}{|l|}{ (B) Root morphological traits } \\
\hline Root length & $\mathrm{RL}$ & $\mathrm{cm}$ \\
\hline Root surface Area & RSA & $\mathrm{cm}^{2}$ \\
\hline Root volume & RV & $\mathrm{cm}^{3}$ plant $^{-1}$ \\
\hline Root average diameter & RAD & $\mathrm{mm}$ \\
\hline Root length to volume & $\mathrm{L} / \mathrm{V}$ & - \\
\hline Specific root length & SRL & $\mathrm{cm} / \mathrm{g}$ \\
\hline Root mass density & RMD & $\mathrm{g} / \mathrm{cm}^{3}$ \\
\hline Number of tips & \#tips & plant $^{-1}$ \\
\hline Number of forks & \#forks & plant $^{-1}$ \\
\hline Root angle & RA & 0 \\
\hline Root dry weight & RDW & g plant $^{-1}$ \\
\hline Root to shoot ratio & R:S & - \\
\hline \multicolumn{3}{|l|}{ (C) Root anatomical traits } \\
\hline Root cross-section area & RXA & $\mathrm{mm}^{2}$ \\
\hline Late metaxylem number & LMXN & plant $^{-1}$ \\
\hline Average Late metaxylem area & ALMXA & $\mathrm{mm}$ \\
\hline Total cortical area & TCA & $\mathrm{mm}^{2}$ \\
\hline Stele area & SA & $\mathrm{mm}^{2}$ \\
\hline Aerenchyma area & AA & $\mathrm{mm}^{2}$ \\
\hline
\end{tabular}


Table 2

Comparison of organic and conventional population root phenotypes under field and hydroponic evalı

\begin{tabular}{|c|c|c|c|c|c|c|c|c|c|c|c|c|}
\hline \multicolumn{7}{|l|}{ Field } & \multicolumn{6}{|c|}{ Hydroponics } \\
\hline \multirow[t]{2}{*}{ Traits } & \multicolumn{4}{|c|}{ Confidence interval } & \multicolumn{2}{|l|}{ Average } & \multicolumn{2}{|c|}{ Standard deviation } & \multicolumn{4}{|c|}{ Confidence interval } \\
\hline & Difference* & lower & upper & $\begin{array}{l}\text { adj } P \\
\text { value }\end{array}$ & Conventional & Organic & Conventional & Organic & Difference* & lower & upper & $\begin{array}{l}\text { adj } \\
\text { valı }\end{array}$ \\
\hline $\mathrm{RL}$ & 25.260 & -3.177 & 53.697 & 0.101 & 277.949 & 303.209 & 74.652 & 84.043 & 584.480 & 326.313 & 842.647 & 0.00 \\
\hline$L: V$ & 42.621 & 18.875 & 66.367 & 0.000 & 158.340 & 200.961 & 52.986 & 77.172 & 341.173 & 220.528 & 461.818 & 0.00 \\
\hline RMD & 0.065 & 0.009 & 0.121 & 0.015 & 0.270 & 0.335 & 0.117 & 0.188 & -0.003 & -0.035 & 0.029 & 0.99 \\
\hline RA & -4.100 & -7.279 & -0.922 & 0.005 & 90.949 & 86.848 & 6.915 & 10.600 & - & - & - & - \\
\hline RSA & -0.526 & -8.546 & 7.494 & 0.998 & 79.231 & 78.705 & 18.910 & 24.077 & 24.433 & -10.254 & 59.120 & $0.2 \epsilon$ \\
\hline RAD & -0.091 & -0.163 & -0.018 & 0.007 & 0.964 & 0.873 & 0.197 & 0.203 & -0.008 & -0.046 & 0.029 & 0.93 \\
\hline \#Tips & 165.890 & 19.043 & 312.736 & 0.020 & 1046.077 & 1211.967 & 357.145 & 440.106 & - & - & - & - \\
\hline \#Forks & 280.142 & -15.44 & 575.725 & 0.070 & 1903.161 & 2183.303 & 680.414 & 884.413 & - & - & - & - \\
\hline SRL & 59.392 & -41.66 & 160.448 & 0.426 & 642.498 & 701.890 & 234.757 & 316.895 & 1577.189 & -582.9 & 3737.3 & 0.23 \\
\hline $\mathrm{RV}$ & -0.228 & -0.480 & 0.024 & 0.091 & 1.923 & 1.695 & 0.681 & 0.700 & 0.216 & -0.235 & 0.666 & $0.6 c$ \\
\hline RDW & 0.041 & -0.065 & 0.147 & 0.747 & 0.504 & 0.545 & 0.252 & 0.335 & 0.025 & -0.095 & 0.146 & 0.94 \\
\hline $\mathrm{R}: \mathrm{S}$ & - & - & - & - & - & - & - & - & -0.080 & -0.151 & -0.010 & 0.01 \\
\hline SA & -0.009 & -0.027 & 0.009 & 0.537 & 0.230 & 0.221 & 0.042 & 0.057 & 0.002 & -0.013 & 0.017 & $0.9 \subseteq$ \\
\hline AA & -0.054 & -0.093 & -0.016 & 0.002 & 0.341 & 0.287 & 0.110 & 0.099 & -0.005 & -0.006 & -0.004 & $0.0 c$ \\
\hline ALMXA & 0.000 & 0.000 & 0.000 & 0.288 & 0.002 & 0.002 & 0.000 & 0.001 & -0.001 & -0.001 & 0.000 & 0.00 \\
\hline LMN & 0.417 & 0.128 & 0.705 & 0.001 & 4.755 & 5.172 & 0.733 & 0.858 & -0.258 & -0.575 & 0.059 & 0.15 \\
\hline RXA & -0.129 & -0.213 & -0.045 & 0.001 & 1.053 & 0.924 & 0.237 & 0.227 & 0.005 & -0.035 & 0.045 & $0.9 \varepsilon$ \\
\hline TCA & -0.120 & -0.192 & -0.048 & 0.000 & 0.891 & 0.771 & 0.210 & 0.186 & 0.004 & -0.025 & 0.032 & $0.9 \varepsilon$ \\
\hline DE & - & - & - & - & - & - & - & - & 0.951 & 0.511 & 1.392 & 0.00 \\
\hline $\mathrm{PH}$ & - & - & - & - & - & - & - & - & 3.118 & 0.552 & 5.684 & 0.01 \\
\hline LN & - & - & - & - & - & - & - & - & 2.473 & -0.846 & 5.792 & 0.21 \\
\hline $\mathrm{TN}$ & - & - & - & - & - & - & - & - & -0.298 & -1.195 & 0.600 & 0.82 \\
\hline SDW & - & - & - & - & - & - & - & - & 0.312 & 0.011 & 0.612 & $0.0=$ \\
\hline
\end{tabular}

Tukey HSD pairwise comparison for root morphology, anatomy and shoot-related traits at $95 \%$ confidence level showing the difference between group means all root traits (first column) between the two groups of barley offspring lines. Besides, the average value and standard deviation of the values for ISR42-8 and - one for field, the other for hydroponic experiment. Coloring indicates significant traits (gray) and higher average (blue) and standard deviation values (greer to smaller in organic lines. See Table 1 for trait description. 
Table 3

Summary of allometric analysis of root-shoot system traits under hydroponic condition.

\begin{tabular}{|c|c|c|c|c|c|c|c|c|c|c|c|c|c|c|c|c|}
\hline Traits & $\mathrm{DE}$ & TN & $\mathrm{LN}$ & $\mathrm{PH}$ & SDW & RL & RSA & RAD & RV & $\mathrm{L} / \mathrm{V}$ & RDW & SRL & RMD & $R: S$ & RXA & ALMXA \\
\hline DE & 1 & 0.017 & 0.567 & 0.027 & 0.273 & 0.58 & 0.753 & 0 & 0.347 & 0.017 & 0.077 & 0.76 & 0.08 & 0.723 & 0.683 & 0.677 \\
\hline TN & 0.017 & 1 & 0.15 & 1 & 0.88 & 1 & 0.523 & 0.997 & 0.893 & 1 & 1 & 1 & 1 & 1 & 1 & 1 \\
\hline LN & 0.567 & 0.15 & 1 & 1 & 0.897 & 1 & 0.84 & 0.993 & 0.897 & 1 & 0.98 & 1 & 1 & 1 & 1 & 1 \\
\hline TPH & 0.027 & 1 & 1 & 1 & 0 & 0.227 & 0.037 & 0.207 & 0.03 & 0.327 & 0.007 & 0.05 & 0.017 & 0.007 & 0.073 & 0.153 \\
\hline SDW & 0.273 & 0.88 & 0.897 & 0 & 1 & 1 & 0.997 & 0.997 & 1 & 1 & 1 & 1 & 1 & 1 & 0.997 & 1 \\
\hline RL & 0.58 & 1 & 1 & 0.227 & 1 & 1 & 1 & 1 & 1 & 1 & 0.943 & 1 & 0.997 & 1 & 1 & 1 \\
\hline RSA & 0.753 & 0.523 & 0.84 & 0.037 & 0.997 & 1 & 1 & 1 & 0.95 & 1 & 0.977 & 1 & 1 & 1 & 1 & 1 \\
\hline RAD & 0 & 0.997 & 0.993 & 0.207 & 0.997 & 1 & 1 & 1 & 0.853 & 0.517 & 0.923 & 0.83 & 0.687 & 0.863 & 0.73 & 0.623 \\
\hline RV & 0.347 & 0.893 & 0.897 & 0.03 & 1 & 1 & 0.95 & 0.853 & 1 & 1 & 0.913 & 1 & 1 & 1 & 1 & 1 \\
\hline L/V & 0.017 & 1 & 1 & 0.327 & 1 & 1 & 1 & 0.517 & 1 & 1 & 1 & 0.973 & 1 & 1 & 1 & 1 \\
\hline RDW & 0.077 & 1 & 0.98 & 0.007 & 1 & 0.943 & 0.977 & 0.923 & 0.913 & 1 & 1 & 1 & 1 & 1 & 1 & 1 \\
\hline SRL & 0.76 & 1 & 1 & 0.05 & 1 & 1 & 1 & 0.83 & 1 & 0.973 & 1 & 1 & 1 & 1 & 1 & 1 \\
\hline RMD & 0.08 & 1 & 1 & 0.017 & 1 & 0.997 & 1 & 0.687 & 1 & 1 & 1 & 1 & 1 & 1 & 1 & 1 \\
\hline $\mathrm{R}: \mathrm{S}$ & 0.723 & 1 & 1 & 0.007 & 1 & 1 & 1 & 0.863 & 1 & 1 & 1 & 1 & 1 & 1 & 1 & 1 \\
\hline RXA & 0.683 & 1 & 1 & 0.073 & 0.997 & 1 & 1 & 0.73 & 1 & 1 & 1 & 1 & 1 & 1 & 1 & 0.9 \\
\hline ALMXA & 0.677 & 1 & 1 & 0.153 & 1 & 1 & 1 & 0.623 & 1 & 1 & 1 & 1 & 1 & 1 & 0.9 & 1 \\
\hline TCA & 0.47 & 1 & 1 & 0.077 & 1 & 1 & 1 & 0.507 & 1 & 1 & 1 & 1 & 1 & 1 & 0.05 & 0.853 \\
\hline$A A$ & 0.717 & 1 & 1 & 0.24 & 1 & 1 & 1 & 0.47 & 1 & 1 & 1 & 1 & 1 & 1 & 0.843 & 0.133 \\
\hline SA & 0.533 & 1 & 1 & 0.173 & 1 & 1 & 1 & 0.933 & 1 & 1 & 1 & 1 & 1 & 1 & 0.82 & 0.927 \\
\hline
\end{tabular}

The $P$-values of allometric cross comparison of root morphological, anatomical and shoot traits. $P$-value $<0.05$ indicate a significant allometric relation betw by bootstrapping

(boot $=300)$. See Table 1 for traits description.

Table 4

Summary of allometric analysis of root-shoot system traits under field condition.

\begin{tabular}{|c|c|c|c|c|c|c|c|c|c|c|c|c|c|c|c|c|}
\hline Traits & RL & RSA & RAD & RV & $\mathrm{L} / \mathrm{V}$ & RA & RDW & SRL & RMD & \#Tips & \#forks & RXA & ALMXA & TCA & AA & SA \\
\hline RL & 1 & 0.287 & 0.323 & 0.253 & 0.133 & 0 & 0.803 & 0.77 & 0.217 & 0.607 & 0.23 & 0.893 & 0.857 & 0.943 & 0.867 & 0.887 \\
\hline RSA & 0.287 & 1 & 0.017 & 0.377 & 0.11 & 0.153 & 0.597 & 0.607 & 0.053 & 0.303 & 0.327 & 0.773 & 0.84 & 0.797 & 0.83 & 0.907 \\
\hline RAD & 0.323 & 0.017 & 1 & 0.743 & 0.287 & 0.867 & 0.79 & 0.783 & 0.38 & 0.907 & 0.28 & 0.183 & 0.253 & 0.277 & 0.797 & 0.79 \\
\hline RV & 0.253 & 0.377 & 0.743 & 1 & 0.05 & 1 & 0.987 & 0.81 & 0.913 & 1 & 0.993 & 0.977 & 0.98 & 0.957 & 1 & 0.987 \\
\hline L/V & 0.133 & 0.11 & 0.287 & 0.05 & 1 & 0.963 & 0.99 & 0.997 & 0.847 & 0.923 & 0.97 & 0.863 & 0.993 & 0.85 & 0.91 & 0.987 \\
\hline RA & 0 & 0.153 & 0.867 & 1 & 0.963 & 1 & 0 & 0.033 & 0.007 & 0.08 & 0.03 & 0.21 & 0.21 & 0.253 & 0.167 & 0.657 \\
\hline RDW & 0.803 & 0.597 & 0.79 & 0.987 & 0.99 & 0 & 1 & 0.867 & 0.24 & 0.943 & 0.35 & 1 & 1 & 1 & 1 & 1 \\
\hline SRL & 0.77 & 0.607 & 0.783 & 0.81 & 0.997 & 0.033 & 0.867 & 1 & 0.713 & 0.893 & 0.7 & 1 & 1 & 1 & 1 & 1 \\
\hline RMD & 0.217 & 0.053 & 0.38 & 0.913 & 0.847 & 0.007 & 0.24 & 0.713 & 1 & 1 & 0.97 & 1 & 1 & 1 & 1 & 1 \\
\hline \#Tips & 0.607 & 0.303 & 0.907 & 1 & 0.923 & 0.08 & 0.943 & 0.893 & 1 & 1 & 0.823 & 0.947 & 0.94 & 0.963 & 0.967 & 0.667 \\
\hline \#forks & 0.23 & 0.327 & 0.28 & 0.993 & 0.97 & 0.03 & 0.35 & 0.7 & 0.97 & 0.823 & 1 & 1 & 1 & 1 & 1 & 1 \\
\hline RXA & 0.893 & 0.773 & 0.183 & 0.977 & 0.863 & 0.21 & 1 & 1 & 1 & 0.947 & 1 & 1 & 0.463 & 0.12 & 0.977 & 0.047 \\
\hline ALMXA & 0.857 & 0.84 & 0.253 & 0.98 & 0.993 & 0.21 & 1 & 1 & 1 & 0.94 & 1 & 0.463 & 1 & 0.927 & 0.983 & 0.387 \\
\hline TCA & 0.943 & 0.797 & 0.277 & 0.957 & 0.85 & 0.253 & 1 & 1 & 1 & 0.963 & 1 & 0.12 & 0.927 & 1 & 0.803 & 0.033 \\
\hline AA & 0.867 & 0.83 & 0.797 & 1 & 0.91 & 0.167 & 1 & 1 & 1 & 0.967 & 1 & 0.977 & 0.983 & 0.803 & 1 & 0.3 \\
\hline SA & 0.887 & 0.907 & 0.79 & 0.987 & 0.987 & 0.657 & 1 & 1 & 1 & 0.667 & 1 & 0.047 & 0.387 & 0.033 & 0.3 & 1 \\
\hline
\end{tabular}

The $P$-values of allometric cross comparison of root morphological, anatomical and shoot traits. $P$-values $<0.05$ indicate a significant allometric relation between the traits calculated by bootstrapping (boot $=300)$. See Table 1 for traits description. 

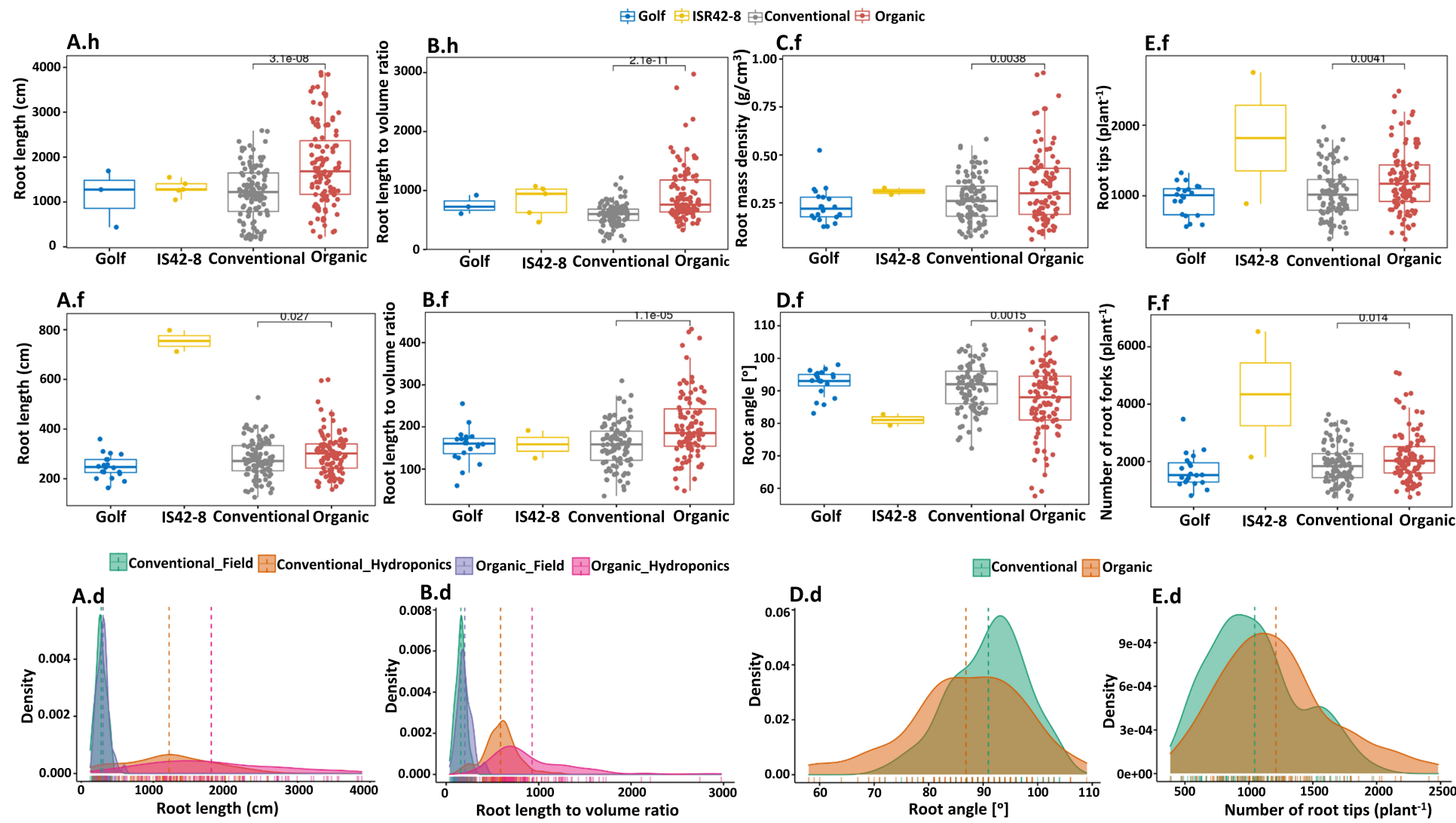

Figure 1

Significantly variant root morphological phenotypes. Boxplots illustrate the overall distribution of observed data points for the parents Golf and ISR $42-8$ as well as for the conventional (CCS) and organic (OCS) lines. Density plots highlight the overall distribution of organic and conventional adapted lines. A - Root length (RL) - sum of all roots harvested in milli meters $(\mathrm{mm})$, illustrated for all four groups. A.h - root length measured in hydroponic experiment; A.f - field experiment; A.d - distribution histogram for root length in both field and hydroponic experiment for CCS and OCS adapted lines. B - the ratio of root length to volume (L/V). Data available for hydroponics (B.h), field (B.f) and distribution of the ratio of root length to volume illustrated in B.d. C.f - Root mass density (RMD) from field; D.f - Root angle (RA) from field, distribution of the root angle illustrated in D.d; E.f - root tip per plant count from field, corresponding histrogram visualized in E.d. F.f - root fork per plant count from field.

\section{Figure 2}

Significantly variant root anatomical traits. Boxplots illustrate the overall distribution of observed data points for the parents Golf and ISR $42-8$ as well as for the conventional (CCS) and organic (OCS) lines. A - Late metaxylem number (LMN) - sum of all roots harvested and expressed by plant ${ }^{-1}$, illustrated for all four groups. A.h - Late metaxylem number measured in hydroponic experiment; A.f - field experiment; A.d - distribution histogram for late metaxylem number in both field and hydroponic experiment for CCS and OCS adapted lines. B - Aerenchyma area (AA). Data available for hydroponics (B.h), field (B.f) and distribution of the aerenchyma area illustrated in B.d. C.f - Total cortical area (TCA) from field; D.f - Root cross-section area (RA) from field, distribution of the total cortical area and root cross-section area illustrated in C.d and D.d, respectively.

\section{Figure 3}

Above-ground plant characteristics. Boxplots illustrate the overall distribution of observed data points for the parents Golf and ISR $42-8$ as well as for the conventional (CCS) and organic (OCS) lines under hydroponic experiment. A- Leaf number (LN) expressed by; B- Plant height (PH) and C-Shoot dry weight (SDW). The data distribution of the leaf number, plant height and shoot dry weight illustrated in D, E and F, respectively. 
A.h

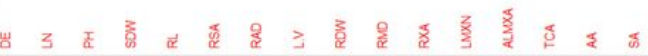

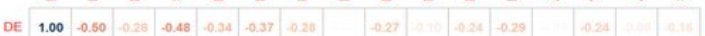

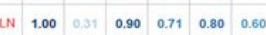

\begin{tabular}{ll|l|l|l|l}
\hline PH & 1.00 & 0.42 & 0.44 & 0.45 & 0.32
\end{tabular}

\begin{tabular}{l|l|l|l|l}
\hline SDW & 1.00 & 0.72 & 0.82 & 0.58
\end{tabular}

\begin{tabular}{lllll}
\hline R. & $100 \quad 0.91$ & 0.54
\end{tabular}

\begin{tabular}{l|l|l|l|l|l|l|l|l} 
RSA & 1.00 & 0.58
\end{tabular}

RAD 1.00

LV 1.00

RDW $1.00 \quad 0.7$

$$
\text { RMD } 1.00
$$

RXA 100

$$
\text { IMXN }
$$

A.f

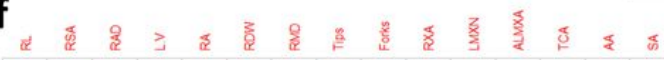

RSA

RAD 1.00

LV $1.00=0.44$

$\begin{array}{llll}-0.78 & -0.77 & -0.85\end{array}$

\begin{tabular}{l|l|l|l|l|} 
ROW & 1.00 & 0.76 & 0.78 & 0.90
\end{tabular}

\begin{tabular}{l|l|l|l} 
RMD & 1.00 & 0.65 & 0.66
\end{tabular}

\begin{tabular}{l|l|l|} 
Tips & 1.00 & 0.83
\end{tabular}

Forks 1.00

RXA 1.00

$$
\text { LMXN } 1.00
$$

\begin{tabular}{ll|l|l|}
0.99 & 0.86 & 0.71
\end{tabular}

ALMXA 1.00

TCA

TCA

SA 1.00

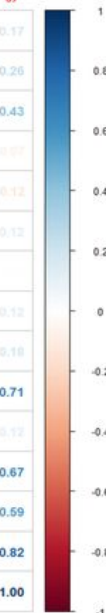

B.h

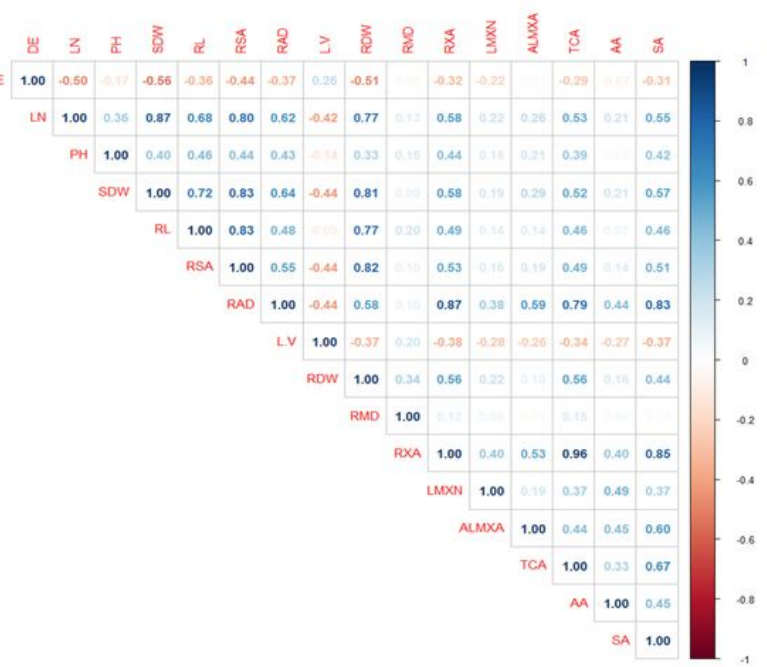

\section{B.f}

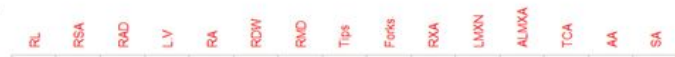

\begin{tabular}{l|l|l|l|l|l|l|l|l|l|}
\hline RL & 1.00 & 0.66 & -025 & 0.34 & -0.91 & 0.85 & 0.70 & 0.74 & 0.84 \\
\hline
\end{tabular}

\begin{tabular}{l|l|l|l|l|l|l|l|l|l|l|l|l|l}
\hline RSA & 1.00 & 0.46 & -0.36 & -0.62 & 0.77 & 0.21 & 0.75 & 0.87 \\
\hline
\end{tabular}

\begin{tabular}{l|l}
1.00 & -0.89 \\
L.V & 1.00
\end{tabular}

RA

\begin{tabular}{lll|l|l|l|}
\hline 1.00 & -0.81 & -0.65 & -0.72 & -0.77
\end{tabular}

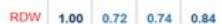

\begin{tabular}{l|l|l|l|} 
RMD & 1.00 & 0.47 & 0.48
\end{tabular}

Tips $1.00 \quad 0.86$

Forks 1.00

RXA 1.00

IMXN

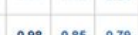

\begin{tabular}{|l|l|l|l|l|}
\hline ALMMXA & 1.00 & 0.44 & 0.57 & 0.60 \\
\hline
\end{tabular}

\begin{tabular}{|l|l|l|l|}
\hline TCA & 1.00 & 0.80 & 0.64 \\
\hline
\end{tabular}

\begin{tabular}{|l|l|l|}
\hline AA & 1.00 & 0.75 \\
\hline
\end{tabular}

SA 1.00

Figure 4

Correlation matrix for shoot morphological (only in hydroponic conditions; A.h and B.h), root architectural and anatomical traits in two groups of barley populations and their parental lines grown across two growing conditions. A- conventional and B- organic cropping systems. A.h-conventional under hydroponic, B.h- organic under hydroponic, A.f- conventional under field and B.f- organic under field conditions. Color scale represents Spearman's ranked correlation coefficient. Larger circle size indicates smaller $p$-value; blank cells represent that correlation was non-significant at $P<0.05$. Most strongly associated traits are ordered and grouped in black boxes based on hierarchical clustering. See Table 1 for trait description.

Figure 5

The principal component output of the root morphology assessment in a hydroponic (A) and a field-based environment (B). The first two components are plotted, with the first PC on the $x$-axis and the second PC on the $y$-axis. The colours differentiate the conventional (magenta) and organic (purple) systems, and the wild donor (ISR 42-8, turquoise) from the cultivar (Golf, green). Each point represents an individual genotype. In ( $A$ ), 150 genotypes were tested from the organic and conventional systems, while 100 genotypes each were tested in (B). Dashed ellipses are plotted based on the points if the sample size was sufficient. Both experiments illustrate higher genetic variance in the organic system as compared to the conventional system.

\section{Supplementary Files}

This is a list of supplementary files associated with this preprint. Click to download.

- Supplementaryinformation.pdf 